\title{
Preoperative cerebral blood flow is diminished in neonates with severe congenital heart defects
}

\author{
Daniel J. Licht, MD, Jiongjiong Wang, PhD, ${ }^{\mathrm{b}}$ David W. Silvestre, BS, ${ }^{a}$ Susan C. Nicolson, MD, ${ }^{\mathrm{c}}$ \\ Lisa M. Montenegro, MD, ${ }^{c}$ Gil Wernovsky, MD, ${ }^{\mathrm{c}, \mathrm{d}}$ Sarah Tabbutt, MD, PhD, ${ }^{\mathrm{c}, \mathrm{d}}$ Suzanne M. Durning, RRT, \\ David M. Shera, ScD, J. William Gaynor, MD, ${ }^{g}$ Thomas L. Spray, MD, ${ }^{9}$ Robert R. Clancy, MD, ${ }^{a}$ \\ Robert A. Zimmerman, $M D^{h}$ and John A. Detre, $M D^{i}$
}

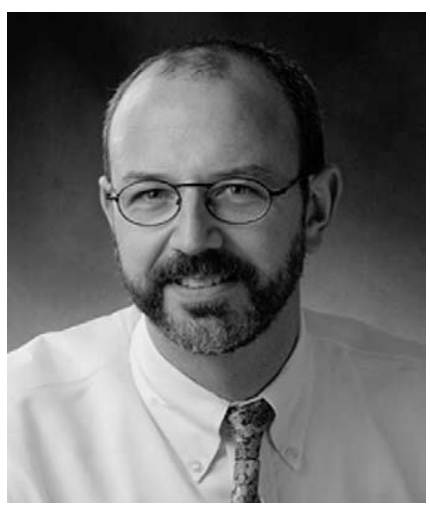

Dr Licht

From the Division of Neurology, ${ }^{a}$ the Division of Anesthesiology and Critical Care, ${ }^{c}$ the Division of Cardiology, ${ }^{\mathrm{d}}$ the Division of Respiratory Therapy, ${ }^{\mathrm{e}}$ the Division of Biostatistics and Epidemiology, ${ }^{\mathrm{f}}$ the Division of Cardiothoracic Surgery, ${ }^{\text {g }}$ and the Division of Neuroradiology, ${ }^{\mathrm{h}}$ The Children's Hospital of Philadelphia, Philadelphia, Pa; and the Divisions of Radiology and Neurology, ${ }^{\mathrm{i}}$ The Hospital of the University of Pennsylvania, Philadelphia, Pa.

This work was completed while Dr Licht was a Pfizer Scholar who was also supported by a grant from the W. W. Smith Charitable Trust. Dr. Wang was supported by the Thrasher Fund for pediatric research.

Read at the Eighty-fourth Annual Meeting of The American Association for Thoracic Surgery, Toronto, Ontario, Canada, April 25-28, 2004.

Received for publication April 23, 2004; revisions received July 12, 2004; accepted for publication July 19, 2004

Address for reprints: Daniel Licht, MD, The Children's Hospital of Philadelphia, Department of Pediatrics, Division of Neurology, Wood Bldg, 6th Floor, 34th and Civic Center Blvd, Philadelphia, PA 19104. (E-mail: licht@email.chop.edu).

J Thorac Cardiovasc Surg 2004;128:841-9 $0022-5223 / \$ 30.00$

Copyright (C) 2004 by The American Association for Thoracic Surgery

doi:10.1016/j.jtcvs.2004.07.022
Objective: Impaired neurodevelopmental outcome represents a major morbidity for survivors of infant heart surgery for congenital heart defects. Previous studies in these neonates have reported preoperative microcephaly, periventricular leukomalacia, and other findings. The hypothesis of this study is that preoperative cerebral blood flow is substantially diminished and might relate to preoperative neurologic conditions.

Methods: Preoperative brain magnetic resonance imaging was performed. Cerebral blood flow measurements in infants with congenital heart defects were obtained by using a novel noninvasive magnetic resonance imaging technique, pulsed arterial spin-label perfusion magnetic resonance imaging. Cerebral blood flow was measured before the operation under standard ventilation and repeated after increased carbon dioxide.

Results: A total of 25 term infants were studied. The average age at the time of the operation was $4.4 \pm 4.6$ days. Congenital heart defects varied widely. Microcephaly occurred in 24\% (6/25). Baseline cerebral blood flow was $19.7 \pm$ $9.2 \mathrm{~mL} \cdot 100 \mathrm{~g}^{-1} \cdot \min ^{-1}\left(8.0-42.2 \mathrm{~mL} \cdot 100 \mathrm{~g}^{-1} \cdot \mathrm{min}^{-1}\right)$. Five patients had cerebral blood flow measurements of less than $10 \mathrm{~mL} \cdot 100 \mathrm{~g}^{-1} \cdot \mathrm{min}^{-1}$. Mean hypercarbic cerebral blood flow increased to $40.1 \pm 20.3 \mathrm{~mL} \cdot 100 \mathrm{~g}^{-1} \cdot \mathrm{min}^{-1}$ $\left(11.4-94.0 \mathrm{~mL} \cdot 100 \mathrm{~g}^{-1} \cdot \min ^{-1}, P<.001\right)$. Pairwise analyses found that low hemoglobin levels were associated with higher baseline cerebral blood flow values $(P=.04)$. Periventricular leukomalacia occurred in $28 \%(7 / 25)$ and was associated with decreased baseline cerebral blood flow values $(P=.05)$ and a smaller change in cerebral blood flow with hypercarbia $(P=.003)$.

Conclusions: Structural brain abnormalities are common in these neonates before surgical intervention. Preoperative cerebral blood flow for this cohort was low and drastically reduced in some patients. Low cerebral blood flow values were associated with periventricular leukomalacia. Carbon dioxide reactivity was preserved but might be compromised by some aspects of the cardiac anatomy. The full spectrum of cerebral blood flow measurements with this technique in congenital heart defects and their long-term significance require continued investigation.

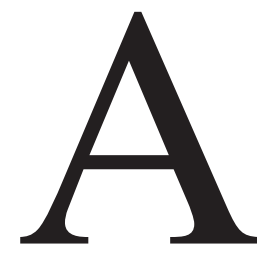

pproximately 30,000 infants are born with congenital heart defects (CHD) in the United States each year. A third of these infants will have severe and complex cardiac lesions that will require surgical repair in the first few months of life. Increased survival after neonatal cardiac surgery has since shifted physicians' attention to preventing neurologic injury and improving neurocognitive outcome in these high-risk patients. ${ }^{1-5}$ Studies of preoperative risk factors in this patient population are extremely limited. 
Mahle and colleagues ${ }^{6}$ performed preoperative and postoperative brain magnetic resonance imaging (MRI) examinations and MRI spectroscopy in infants with complex CHDs and found preoperative periventricular leukomalacia (PVL) in 4 (17\%) of 24 infants. Furthermore, 10 (53\%) of 19 infants who underwent preoperative spectroscopy showed increased white matter lactate levels, suggesting tissue ischemia. The proportion of patients with PVL determined on the basis of postoperative MRI increases to more than $50 \% .^{7}$ Reported autopsy findings in 39 neonates with hypoplastic left heart syndrome showed a high occurrence of microencephaly (low brain weight) and open opercula, a minor form of cortical underdevelopment, which suggest chronic cerebral underperfusion. ${ }^{8,9}$

PVL principally arises from injury to immature oligodendrocytes, which are particularly prone to ischemia ${ }^{10}$ in cerebral watershed areas. ${ }^{11}$ In preterm infants PVL is highly associated with long-term neurodevelopmental consequences, but the significance of PVL in term infants with CHD remains under investigation. Nevertheless, the occurrence of neurodevelopmental sequelae after infant heart surgery is well established. In prospective cohort studies of cognitive outcome after the Fontan procedure, it has been observed that $8 \%$ to $15 \%$ of survivors had full-scale IQs of less than 70. ${ }^{3,4}$

Because a significant proportion of neonates with $\mathrm{CHD}$ have PVL, microcephaly, or underdeveloped opercula before heart surgery, their presence requires an examination of preoperative factors, such as cerebral blood flow (CBF), that might be related to short-term and long-term central nervous system (CNS) consequences. To our knowledge, quantitative measurements of CBF exclusively in neonates with CHDs before surgical intervention have never been published. These findings might greatly contribute to our understanding of cerebral injury and might suggest future neuroprotective strategies. This investigation uses pulsed arterial spin-labeling perfusion magnetic resonance imaging (PASL-pMRI) to quantify preoperative CBF. This novel imaging technique uses electromagnetically spin-labeled arterial blood water as a noninvasive diffusible tracer to measure CBF flow in standard physiologic units in milliliters per 100 grams per minute. ${ }^{12}$

The major aims of this study are (1) to investigate the evidence of preoperative CNS anatomic abnormalities, including PVL, in neonates with complex CHDs; (2) to measure preoperative CBF by using PASL-pMRI to test the hypothesis that $\mathrm{CBF}$ is low and might be associated with observed anatomic CNS abnormalities; and (3) to measure cerebral vascular responsiveness to inspired $\mathrm{CO}_{2}$, a known cerebral vasodilator. This is of interest because impaired $\mathrm{CO}_{2}$ reactivity has been associated with poor neurodevelopmental outcome and a higher risk of death in all age groups. ${ }^{13-15}$

\section{Methods}

\section{Patient Population}

With institutional review board approval, all newborn infants with CHDs admitted to the cardiac intensive care unit at The Children's Hospital of Philadelphia were evaluated for study inclusion. Inclusion criteria included full-term age (gestational age, $40 \pm 4$ weeks), an intention to undergo surgical intervention with cardiopulmonary bypass with or without deep hypothermic circulatory arrest (DHCA), and medical stability for 24 hours before the operation. Infants were excluded if they had a history of birth asphyxia (5-minute Apgar score of $<5$ or cord $\mathrm{pH}$ of $<7.0$ ) or a preoperative cardiac arrest requiring chest compressions. Patients with hypoplastic left heart syndrome treated with hypercarbia for pulmonary overcirculation were also excluded.

\section{CHD Anatomies}

A wide variety of specific CHDs were studied (Table 1). Lesions were further anatomically classified into one of 4 categories, as described by Clancy and associates ${ }^{16}$ : additional descriptors, such as an obstructed aortic arch, patent ductus arteriosus, or singleventricle physiology, were included to assess associations with $\mathrm{CBF}$ and $\mathrm{CO}_{2}$ reactivity.

\section{MRI and Perfusion Sequences}

All MRI scans were performed on Siemens Vision or Sonata 1.5T scanners. The full technical details of the imaging and perfusion studies are available elsewhere. ${ }^{12}$ The pulsed arterial spin-labeled MRI sequence (PASL-pMRI) was a modification of a previously described technique. ${ }^{17}$ PASL-pMRI has been compared with gadolinium bolus perfusion MRI in cases of adult stroke ${ }^{18}$ and has been validated in adults against $\mathrm{H}_{2}{ }^{15} \mathrm{O}$ positron emission tomography. ${ }^{19}$ Three PASL-pMRI scans were carried out immediately before the operation under standard ventilator settings, followed by 2 PASL-pMRI scans after increased $\mathrm{CO}_{2}$.

The structural scans included sagittal T1-weighted turbo-spin echocardiography and axial T1, T2, and fluid attenuation inversion recovery sequences. Structural image interpretation was performed by a single neuroradiologist (RAZ), who was blinded to the patient's clinical condition and diagnosis. MRI scans were reviewed for congenital abnormalities and acquired abnormalities, such as PVL, which was graded on a 4-point scale from absent to mild, moderate, or severe. Closure of the operculum was rated as complete or incomplete.

\section{Ventilation and Patient Monitoring}

All patients had indwelling umbilical or peripheral arterial and venous catheters that were not dictated by study participation. All patients were mechanically ventilated and stabilized with a baseline goal $\mathrm{PaCO}_{2}$ of $40 \mathrm{~mm} \mathrm{Hg}$ confirmed by means of arterial blood gas analysis before the first $\mathrm{CBF}$ measurement. Hypercarbic gas mixture was administered and adjusted to a goal inspired fraction of $\mathrm{CO}_{2}\left(\mathrm{FiCO}_{2}\right)$ of $20 \mathrm{~mm} \mathrm{Hg}$ (equivalent to an $\mathrm{FiCO}_{2}$ of $2.7 \%$ ). Vital signs and pulse oximetry were monitored continuously.

Prostaglandin infusions were discontinued 1 hour before study initiation. Patients were medicated with fentanyl $(5 \mu \mathrm{g} / \mathrm{kg})$ and pancuronium $(0.2 \mathrm{mg} / \mathrm{kg})$. None received inotropes during the study or boluses of calcium, bicarbonate, or volume expanders. 
TABLE 1. Patient demographics and cardiac diagnoses

\begin{tabular}{lr}
\hline Patient demographics & $\mathbf{N}$ \\
\hline Cohort size & 25 \\
Sex & 13 \\
Male & 12 \\
Female & \\
Race & 3 \\
African American & 17 \\
White & 4 \\
Hispanic & 1 \\
Other & \\
Cardiac diagnosis & 1 \\
Aortic stenosis & 1 \\
Heterotaxy & 7 \\
HLHS & 2 \\
HLHS variant & 2 \\
Single ventricle: other & 1 \\
PA/IVS & 1 \\
TAPVR & 2 \\
TGA/IVS & 2 \\
TGA/VSD & 1 \\
TGA/VSD/coarct & 1 \\
TOF & 1 \\
TOF/PA & 1 \\
Truncus arteriosus & 2 \\
VSD/coarct & \\
\hline &
\end{tabular}

HLHS, Hypoplastic left heart syndrome; $P A$, pulmonary atresia; IVS, intact ventricular septum; TAPVR, total anomalous pulmonary venous return; $T G A$, transposition of the great arteries; VSD, ventricular septal defect; coart, coarctation of the aorta; TOF, tetralogy of Fallot.

\section{Conduct of the Study}

Study patients were transported by the cardiac anesthesia team from the cardiac intensive care unit to the operating room for induction of anesthesia, endotracheal intubation, and establishment of vascular access 1 hour before the scheduled operation. Patients were then transported to the MRI suite. Initial arterial and venous blood samples were drawn for baseline $\mathrm{PaCO}_{2}$ measurement. On completion of the first perfusion MRI sequence, supplemental $\mathrm{CO}_{2}$ was administered to achieve a goal $\mathrm{FiCO}_{2}$ of $20 \mathrm{~mm} \mathrm{Hg}$. Sequences for structural MRIs were obtained during $\mathrm{CO}_{2}$ equilibration. A second pMRI sequence was then performed to measure CBF in the hypercarbic state. Finally, a second set of blood gas samples was obtained to confirm the higher $\mathrm{PaCO}_{2}$ level. The hypercarbic gas mixture was then discontinued, and the patient was transported directly to the operating room. The average study duration was $83 \pm 6$ minutes.

\section{Statistical Analysis}

Patient and MRI data were characterized by simple descriptive statistics, including mean, SD, median, and range values. Outcome end points were associated with other variables that could be plausibly considered as predictors in a pairwise fashion. For continuous scale predictors, the Pearson and Spearman correlations were examined. Generalized linear models based on many predictor variables were also explored. Because of the limited sample size, not every candidate predictor could be included in every
TABLE 2. Patient demographics

\begin{tabular}{lcc}
\hline & Average & Range \\
\hline Gestational age (wk) & $38.7 \pm 1.2$ & $36-40$ \\
Day of life (d) & $4.7 \pm 4.9$ & $1-26$ \\
Head circumference $(\mathrm{cm})$ & $33.3 \pm 1.2$ & $30.5-30.3$ \\
Birth weight $(\mathrm{kg})$ & $3.1 \pm 0.42$ & $2.5-4$ \\
\hline
\end{tabular}

TABLE 3. Vital signs and blood gas measurements for both baseline and hypercapnic conditions

\begin{tabular}{|c|c|c|c|}
\hline & Baseline $(n=25)$ & Hypercapnic $(n=25)$ & $P$ value* \\
\hline HR & $138.4 \pm 17.3$ & $141.8 \pm 17.7$ & .09 \\
\hline MAP & $55.4 \pm 10.7$ & $56.8 \pm 9.1$ & .75 \\
\hline $\mathrm{FiCO}_{2}$ & $0 \pm 0.0$ & $23.5 \pm 4.5$ & $<.01$ \\
\hline $\mathrm{Hb}$ & $14.6 \pm 1.8$ & $14.6 \pm 1.9$ & .9 \\
\hline $\mathrm{pH}$ & $7.38 \pm 0.06$ & $7.23 \pm 0.06$ & $<.01$ \\
\hline $\mathrm{PaCO}_{2}$ & $40.7 \pm 5.4$ & $61.6 \pm 7.0$ & $<.01$ \\
\hline $\mathrm{PaO}_{2}$ & $43.7 \pm 12.6$ & $52.8 \pm 12.4$ & .0002 \\
\hline $\mathrm{SaO}_{2}$ & $74.2 \pm 14.0$ & $74.5 \pm 14.0$ & .9 \\
\hline $\mathrm{CaO}_{2}$ & $14.6 \pm 3.3$ & $14.7 \pm 3.1$ & .9 \\
\hline
\end{tabular}

$H R$, Heart rate; $M A P$, mean arterial pressure; $\mathrm{FiCO}_{2}$, fraction of inspired carbon dioxide; $\mathrm{Hb}$, hemoglobin concentration; $\mathrm{PaCO}_{2}$, arterial partial pressure of carbon dioxide; $\mathrm{PaO}_{2}$, arterial partial pressure of oxygen; $\mathrm{SaO}_{2}$, arterial oxygen saturation; $\mathrm{CaO}_{2}$, arterial oxygen content.

model. Stepwise selection procedures were conducted to identify strong predictors, which also removed weaker predictors from the model. The stepwise procedure tends to find models that are better than any pairwise model but also more parsimonious than the full model. Finally, some models were examined with interaction terms on the basis of the stepwise models, which shows how one predictor might modify the effect of another.

\section{Results}

\section{Patient Demographics}

The parents of 28 patients were approached for enrollment in this study: of these, 3 declined consent. Of the 25 patients studied, 2 lost CBF data because of a technical difficulty with the MRI. These 2 patients were included for all other analyses of data, including safety analysis and structural MRI data analysis. Tables 1 and 2 describe cardiac diagnoses and patient characteristics, respectively. The cardiac diagnoses were made on the basis of prenatal fetal echocardiography in $13(52 \%)$, at the time of birth in 11 (44\%), and as an outpatient in 1 (4\%).

\section{Hemodynamic and Blood Gas Data}

Table 3 shows the baseline and hypercarbic vital signs, including transcutaneous oxygen saturation, heart rate, and systolic, diastolic, and mean arterial blood pressures. There were no statistically significant changes in vital signs with the administration of $\mathrm{CO}_{2}$ through the ventilator. 


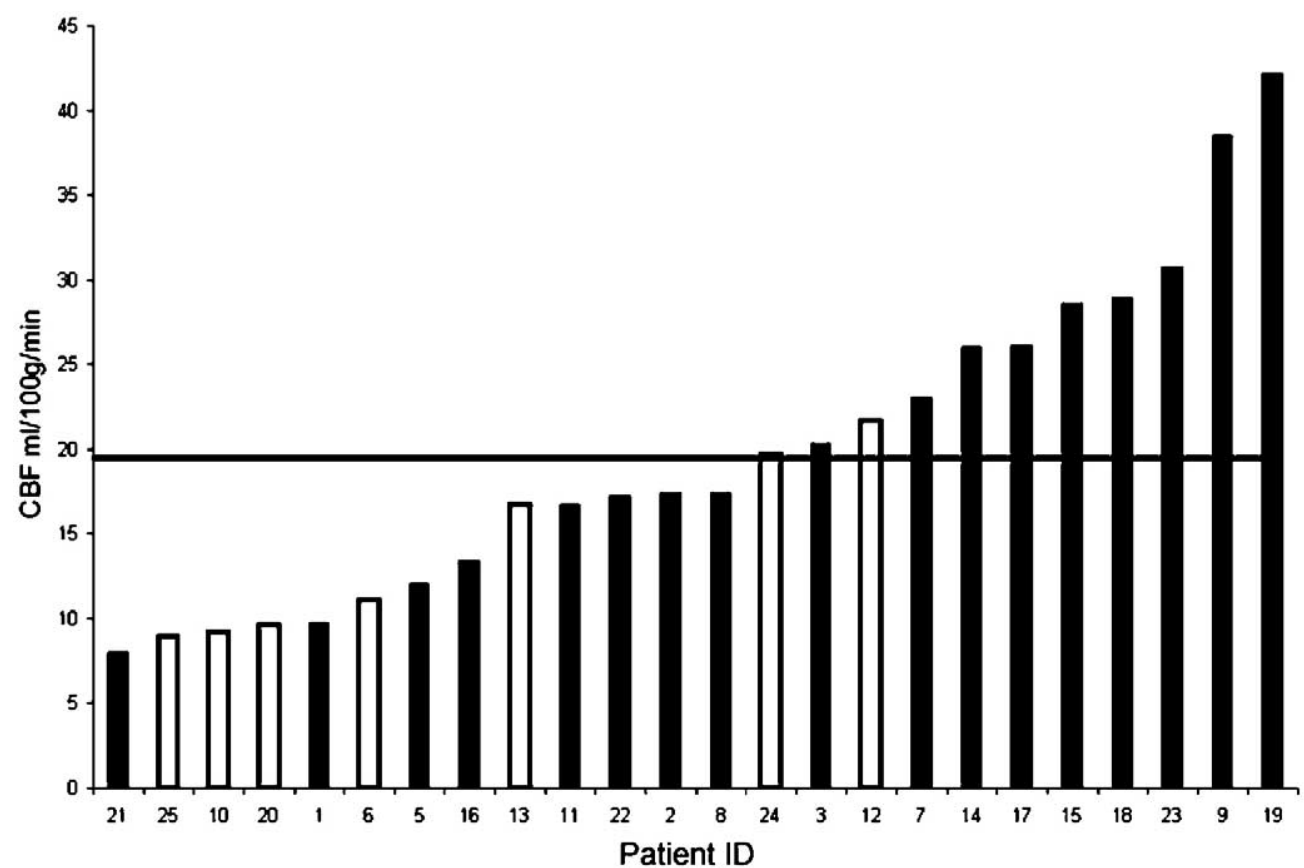

Figure 1. Individual baseline CBF. Black and white bars represent patients with and without PVL, respectively. The heavy horizontal line indicates the average CBF for the cohort.

TABLE 4. Average CBF measurements in patients with and without PVL

\begin{tabular}{|c|c|c|c|}
\hline & Baseline CBF (mL/100 g per min) & Hypercapnic CBF (mL/100 g per minute) & $\mathrm{CO}_{2}$ reactivity $\left(\Delta \mathrm{CBF} / \Delta \mathrm{pCO} \mathrm{O}_{2}\right)$ \\
\hline Total no. of patients & 24 & 23 & 23 \\
\hline All patients & $19.73 \pm 9.15$ & $40.13 \pm 20.31$ & $0.903 \pm 0.546$ \\
\hline Presence of PVL & & & \\
\hline Patients with PVL ( $\mathrm{n}=7)$ & $13.86 \pm 5.39$ & $28.72 \pm 16.21$ & $0.633 \pm 0.326$ \\
\hline Patients without PVL $(n=17)$ & $\begin{array}{c}22.14 \pm 9.67 \\
P=.05\end{array}$ & $\begin{array}{c}45.12 \pm 20.98 \\
P=.003\end{array}$ & $\begin{array}{c}1.02 \pm 0.604 \\
P=.02\end{array}$ \\
\hline
\end{tabular}

CBF, Cerebral blood flow; $P V L$, periventricular leukomalacia.

\section{Cerebral Blood Flow}

Table 4 illustrates the absolute CBF values for the cohort expressed in milliliters per 100 grams per minute at baseline and during hypercarbic conditions, as well as $\mathrm{CO}_{2}$ reactivity, defined as the change in CBF divided by the change in $\mathrm{pCO}_{2}$. Individual changes in $\mathrm{CBF}$ are shown in Figures 1 and 2. Baseline CBF was inversely and linearly associated with hemoglobin concentration $(P=.04)$. There was no significant association between baseline $\mathrm{CBF}$ and heart defect, regardless of their classification. $\mathrm{CO}_{2}$ reactivity for the group was $0.96 \pm 0.61 \mathrm{~mL} \cdot 100 \mathrm{~g}^{-1} \cdot \min ^{-1} \cdot \mathrm{mm} \mathrm{Hg}^{-1}$ change in $\mathrm{pCO}_{2} \cdot \mathrm{CO}_{2}$ reactivity was correlated with diastolic blood pressure (BP) and inversely correlated with hemoglobin concentration $(\mathrm{Hb})$ and PVL $(P=.001, .02$, and .02 , respectively). $\mathrm{CO}_{2}$ reactivity could be predicted by using the following linear regression: $\mathrm{CO}_{2}$ reactivity $=4.1+0.14$ (Diastolic BP) -0.24 (If PVL) - $0.2(\mathrm{Hb})$.
Classifying the CHD by using the methods described by Clancy and associates ${ }^{16}$ failed to resolve differences in $\mathrm{CO}_{2}$ reactivity. The presence of either aortic arch obstruction or single-ventricle physiology showed marked decreased reactivity; however, wide SDs kept them from reaching significance $(P=.14$ and $P=.18$, respectively).

\section{Structural MRI Data}

Thirteen (53\%) patients had evidence of developmental lesions, acquired lesions, or both, including microcephaly, incomplete closure of the operculum, PVL, and other ischemic lesions. PVL was detected in 7 (28\%) patients and was moderate to severe in $3(12 \%)$ patients. Significantly, the presence of PVL was associated with low baseline CBF values $(P=$ $.05)$ and decreased $\mathrm{CO}_{2}$ reactivity $(P=.02$, Table 4$)$. The average head circumference was significantly lower than that of the normal population without heart defects $(P<.0001)$. 


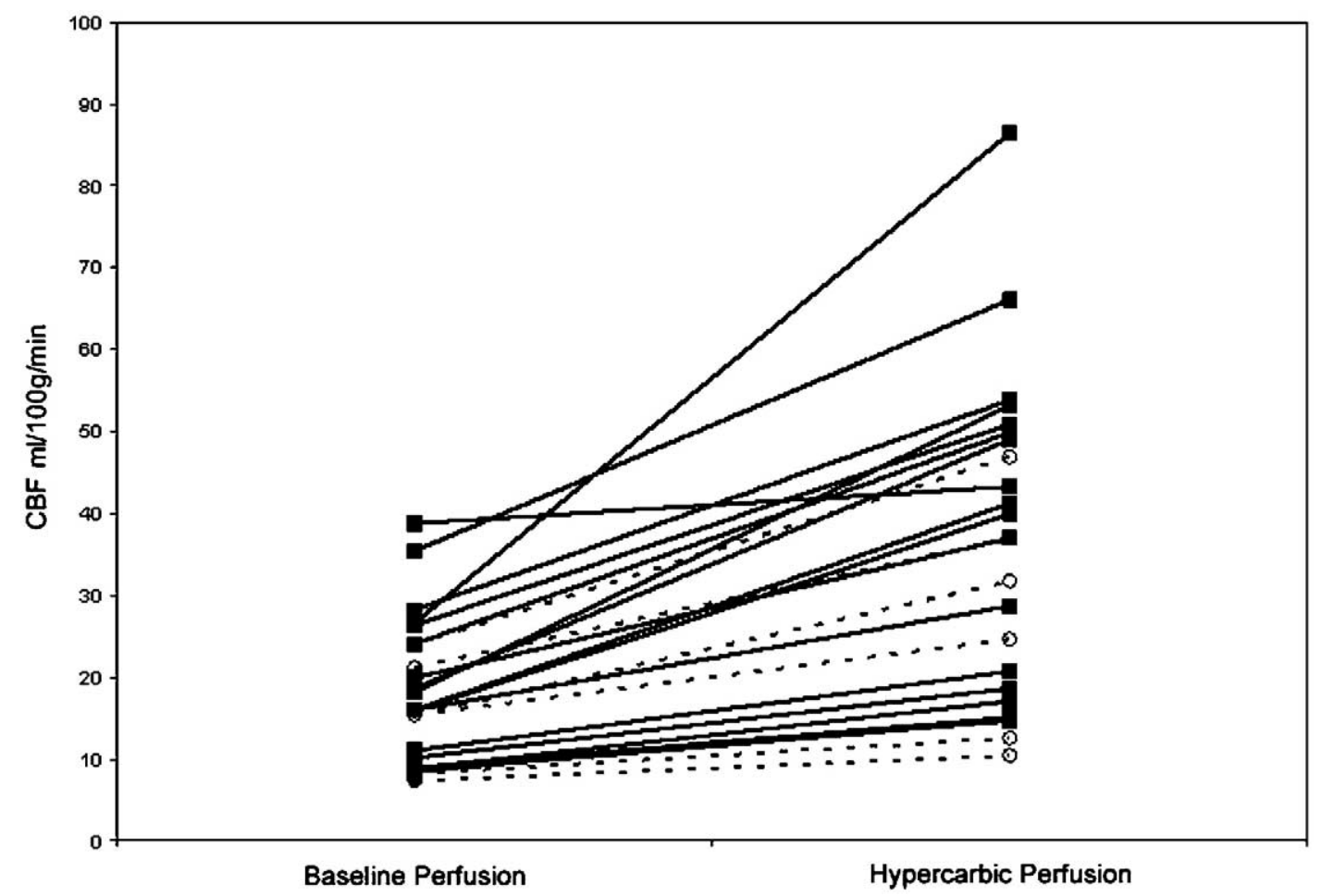

Figure 2. Individual CBF at baseline and with hypercapnic conditions. Solid squares connected by solid black lines denote patients who did not have PVL. Open circles connected by broken lines denote patients with PVL.

Microcephaly, defined as more than 2 SDs less than the normal mean, was seen in $6(24 \%)$ of 25 patients. Incomplete closure of the operculum was identified in $4(16 \%)$ of 25 patients. An association between these 2 structural abnormalities, baseline $\mathrm{CBF}$ and $\mathrm{CO}_{2}$ reactivity, failed to reach statistical significance.

\section{Discussion}

Although there has been wide appreciation of the long-term neurocognitive consequences of neonatal heart surgery, the main focus of attention has been directed to specific intraoperative risk factors, including DHCA, hemodilution, and acid-base management. However, there is a growing body of evidence that suggests that preoperative features might also influence short-term and long-term outcome. The occurrence of preoperative microcephaly, PVL, increased white matter lactate, and the persistence of open opercula raise the concern for chronic cerebral hypoperfusion that might adversely affect the brain long before the conduct of surgical intervention. Specific data on CBF in this population are extremely scarce, and methods for measurement are troublesome. Color Doppler imaging is noninvasive and portable but measures only blood flow velocity, and results are dependent on probe angulation. Near-infrared spectroscopy is also portable and noninvasive but measures changes in cerebral oximetry (not CBF) in only a small area of the cortex. Again, probe placement is crucial. This is the first study using PASL-pMRI to measure global CBF in neonates with severe forms of CHD and to relate these values to observed anatomic abnormalities.

As previously reported in other CHD populations, this study cohort demonstrated several preoperative anatomic abnormalities. ${ }^{6,8,9}$ The most common was PVL, observed in $28 \%$ of the study group. The mean head circumference was significantly less than the norm for term neonates. Microcephaly, an indicator of decreased brain growth and an important marker for adverse neurodevelopment, was the second most common structural brain abnormality and observed in $24 \%$ of the study group. Delayed closure of the opercula occurred in $16 \%$ of the patients. Remarkably, more than $50 \%$ of the study cohort had one or more of these CNS findings that could be attributed to chronic cerebral hypoperfusion.

Prior studies of CBF in pediatric CHD used ${ }^{133} \mathrm{Xe}$ clearance methodology and recorded the effects of hypothermia, cardiopulmonary bypass, and DHCA on CBF and cerebral metabolism. ${ }^{20-23}$ These studies, which included prebypass conditions, examined a broad range of ages that included neonates. In our cohort, CBF values were measured and regarded as specific risk factors for the observed preoperative structural brain abnormalities. The mean CBF value for the cohort was $19.7 \pm$ $9.1 \mathrm{~mL} \cdot 100 \mathrm{~g}^{-1} \cdot \min ^{-1}$, which was much lower than the normal value of $50 \pm 3.4 \mathrm{~mL} \cdot 100 \mathrm{~g}^{-1} \cdot \mathrm{min}^{-1}$ reported by Chiron and coworkers ${ }^{24}$ in term infants under conditions of 
conscious sedation. Significantly, 5 neonates had CBF values of less than $10 \mathrm{~mL} \cdot 100 \mathrm{~g}^{-1} \cdot \min ^{-1}$, the level generally recognized to cause moderate ischemic changes in piglets. ${ }^{25}$ Baseline CBF values varied linearly and inversely with hemoglobin concentration. Likewise, $\mathrm{CO}_{2}$ reactivity was modeled as a function of hemoglobin concentration and diastolic blood pressure. These associations occur in all age groups and in different species. The observed low CBF measurements were also congruent with the study of Greeley and associates, ${ }^{20-22}$ which included neonates, although specific neonatal CBF values were not detailed. Markedly low CBF measurements $(<10$ $\mathrm{mL} \cdot 100 \mathrm{~g}^{-1} \cdot \min ^{-1}$ ) have been made in other studies examining critically ill neonates with normal short-term outcomes. ${ }^{26}$

These low CBF measurements are also consistent with prior reports of preoperative increased white matter lactate levels, ${ }^{6}$ indicating a preischemic state. In this study all 3 infants with moderate-to-severe PVL had CBF values of less than $10 \mathrm{~mL} \cdot 100 \mathrm{~g}^{-1} \cdot \min ^{-1}$. It should be noted that although a significant association between low CBF and PVL was demonstrated, a true causal effect was not. Brain metabolism was not concurrently measured; therefore it is not possible to determine whether the low $\mathrm{CBF}$ values represented an appropriate response to low cerebral metabolism from a white matter ischemic state.

$\mathrm{CO}_{2}$ reactivity for the cohort averaged $0.96 \pm 0.61 \mathrm{~mL}$. $100 \mathrm{~g}^{-1} \cdot \mathrm{min}^{-1} \cdot \mathrm{mm} \mathrm{Hg}^{-1}$, with a wide range from 0.22 to $2.17 \mathrm{~mL} \cdot 100 \mathrm{~g}^{-1} \cdot \mathrm{min}^{-1} \cdot \mathrm{mm} \mathrm{Hg}^{-1}$. In previous pediatric studies $\mathrm{CO}_{2}$ reactivity of greater than $2 \mathrm{~mL} \cdot 100 \mathrm{~g}^{-1} \cdot \min ^{-1}$ - $\mathrm{mm} \mathrm{Hg}^{-1}$ was associated with survival, whereas reactivity of less than $1.0 \mathrm{~mL} \cdot 100 \mathrm{~g}^{-1} \cdot \mathrm{min}^{-1} \cdot \mathrm{mm} \mathrm{Hg}^{-1}$ was associated with death or poor neurologic outcome. ${ }^{14,15}$ In this limited cohort, the broad range of reactivity values and heterogeneous patient population precluded detailed predictor analyses, although the presence of aortic arch obstruction and singleventricle physiology trended toward an association with reduced $\mathrm{CO}_{2}$ reactivity. The wide range of $\mathrm{CO}_{2}$ reactivity values obtained were similar to those measured with ${ }^{133} \mathrm{Xe}$ SPECT in infants undergoing venoarterial extracorporeal membrane oxygenation. $^{26}$

PASL-pMRI is a noninvasive technique to measure CBF directly by using electromagnetically labeled arterial blood water as an endogenous tracer. Over the past decade, methodologies for PASL-pMRI have evolved from feasibility studies into practical use ${ }^{12,27}$ and are now being used in pediatric populations. ${ }^{17}$ Our prior studies demonstrated a substantial improvement in the signal-to-noise ratio of perfusion images in neurologically healthy children compared with healthy adults, ${ }^{17}$ and CBF values were comparable with those obtained by using radioactive measurement techniques. ${ }^{24}$ Moreover, a high reproducibility of $5.3 \%\left(6.2 \mathrm{~mL} \cdot 100 \mathrm{~g}^{-1} \cdot \mathrm{min}^{-1}\right)$ at the $95 \%$ confidence interval was observed for the 2 repeated perfusion scans after $\mathrm{CO}_{2}$ administration. The accuracy of
PASL measurements in the presence of drastically reduced $\mathrm{CBF}$ and slow blood flow requires further evaluation.

The absence of a control group is a major limitation of this study. In light of this limitation, neonates without CHD might undergo CBF determination with PASL-pMRI, but it is acknowledged that these studies will not be acquired under identical conditions as in the neonates with CHDs.

We demonstrate that preoperative structural brain abnormalities were seen in more than $50 \%$ of the cohort, with the most common being PVL (28\%) and microcephaly (24\%). $\mathrm{CBF}$ in these neonates was measured safely and noninvasively with PASL-pMRI. Observed CBF values were much lower than what is suggested by limited normal data and critically decreased in some patients. The presence of PVL was strongly associated with low baseline $\mathrm{CBF}$ and poor $\mathrm{CO}_{2}$ reactivity.

There continue to be important gaps in our knowledge concerning the pathogenesis of adverse neurodevelopment in infants with severe CHD. These data draw attention to the potential role of low CBF being an underrecognized preoperative risk factor for poor neurocognitive outcome. The observation in which hypercarbia increases CBF to near-normal levels provides hope that this or other potential agents might be exploited in future neuroprotection strategies.

We thank Dr R. Ichord, Mr Dongbo Hu, Ms Christine Harris, Mr Ralph Magee, and Mr Erin O'Tool for their cooperation, critical comments, and technical assistance.

\section{References}

1. Mahle WT, Wernovsky GR. Long-term developmental outcome of children with complex congenital heart disease. Clin Perinatol. 2001; 28:235-47.

2. Limperopoulos C, Majnemer A, Shevell MI, Rosenblatt B, Rohlicek $\mathrm{C}$, Tchervenkov Cr. Neurodevelopmental status of newborns and infants with congenital heart defects before and after open heart surgery. J Pediatr. 2000;137:638-45.

3. Wernovsky G, Stiles KM, Gauvreau K, et al. Cognitive development after the Fontan operation. Circulation. 2000;102:883-9.

4. Mahle WT, Clancy RR, Moss EM, Gerdes M, Jobes DR, Wernovsky GR. Neurodevelopmental outcome and lifestyle assessment in schoolaged and adolescent children with hypoplastic left heart syndrome. Pediatrics. 2000;105:1082-9.

5. Bellinger DC, Wernovsky G, Rappaport LA, et al. Cognitive development of children following early repair of transposition of the great arteries using deep hypothermic circulatory arrest. Pediatrics. 1991; 87:701-7.

6. Mahle WT, Tavani F, Zimmerman RA, et al. An MRI study of neurological injury before and after congenital heart surgery. Circulation. 2002;106(suppl II):I109-14.

7. Clancy RR, McGaurn SA, Goin JE, et al. Allopurinol neurocardiac protection trial in infants undergoing heart surgery using deep hypothermic circulatory arrest. Pediatrics. 2001;108:61-70.

8. Glauser TA, Rorke LB, Weinberg PM, Clancy RR. Acquired neuropathologic lesions associated with the hypoplastic left heart syndrome. Pediatrics. 1990;85:991-1000.

9. Glauser TA, Rorke LB, Weinberg PM, Clancy RR. Congenital brain anomalies associated with the hypoplastic left heart syndrome. Pediatrics. 1990;85:984-90.

10. Volpe JJ. Neurobiology of periventricular leukomalacia in the premature infant. Pediatr Res. 2001;50:553-62. 
11. Rorke L. Pathology of perinatal brain injury. New York: Raven Press; 1989.

12. Detre JA, Samuels OB, Alsop DC, Gonzalez-At JB, Kasner SE, Raps EC. Noninvasive magnetic resonance imaging evaluation of cerebral blood flow with acetazolamide challenge in patients with cerebrovascular stenosis. J Magn Reson Imaging. 1999;10:870-5.

13. Pryds O, Greisen G, Skov LL, Friis-Hansen B. Carbon dioxide-related changes in cerebral blood volume and cerebral blood flow in mechanically ventilated preterm neonates: comparison of near infrared spectrophotometry and ${ }^{133}$ Xenon clearance. Pediatr Res. 1990;27:445-9.

14. Ashwal S, Stringer W, Tomasi L, Schneider S, Thompson J, Perkin R. Cerebral blood flow and carbon dioxide reactivity in children with bacterial meningitis. J Pediatr. 1990;117:523-30.

15. Ashwal S, Perkin RM, Thompson JR, Tomasi LG, van Stralen D, Schneider S. CBF and CBF/PCO2 reactivity in childhood strangulation. Pediatr Neurol. 1991;7:369-74.

16. Clancy RR, McGaurn SA, Wernovsky G, et al. Preoperative risk-of-death prediction model in heart surgery with deep hypothermic circulatory arrest in the neonate. J Thorac Cardiovasc Surg. 2000;119:347-57.

17. Wang J, Licht DJ, Jahng GH, et al. Pediatric perfusion imaging using pulsed arterial spin labeling. J Magn Reson Imaging. 2003;18:404-13.

18. Siewert B, Schlaug G, Edelman RR, Warach S. Comparison of EPISTAR and $\mathrm{T} 2 *$-weighted gadolinium-enhanced perfusion imaging in patients with acute cerebral ischemia. Neurology. 1997;48:673-9.

19. Ye FQ, Berman KF, Ellmore T, et al. H(2)(15)O PET validation of steady-state arterial spin tagging cerebral blood flow measurements in humans. Magn Reson Med. 2000;44:450-6.

20. Greeley WJ, Kern FH, Ungerleider RM, et al. The effect of hypothermic cardiopulmonary bypass and total circulatory arrest on cerebral metabolism in neonates, infants, and children. $J$ Thorac Cardiovasc Surg. 1991;101:783-94.

21. Greeley WJ, Ungerleider RM, Smith LR, Reves JG. The effects of deep hypothermic cardiopulmonary bypass and total circulatory arrest on cerebral blood flow in infants and children. $J$ Thorac Cardiovasc Surg. 1989;97:737-45.

22. Greeley WJ, Ungerleider RM, Kern FH, Brusino FG, Smith LR, Reves JG. Effects of cardiopulmonary bypass on cerebral blood flow in neonates, infants, and children. Circulation. 1989;80(suppl I):I209-15.

23. Kern FH, Ungerleider RM, Quill TJ, et al. Cerebral blood flow response to changes in arterial carbon dioxide tension during hypothermic cardiopulmonary bypass in children. $J$ Thorac Cardiovasc Surg. 1991;101:618-22.

24. Chiron C, Raynaud C, Maziere B, et al. Changes in regional cerebral blood flow during brain maturation in children and adolescents. $\mathrm{J} \mathrm{Nucl}$ Med. 1992;33:696-703.

25. Ichord RN, Kirsch JR, Helfaer MA, Haun S, Traystman RJ. Agerelated differences in recovery of blood flow and metabolism after cerebral ischemia in swine. Stroke. 1991;22:626-34.

26. Stockwell JA, Goldstein RF, Ungerleider RM, Kern FH, Meliones JN, Greeley WJ. Cerebral blood flow and carbon dioxide reactivity in neonates during venoarterial extracorporeal life support. Crit Care Med. 1996;24:155-62.

27. Floyd TF, Ratcliffe SJ, Wang J, Resch B, Detre JA. Precision of the CASL-perfusion MRI technique for the measurement of cerebral blood flow in whole brain and vascular territories. J Magn Reson Imaging. 2003;18:649-55.

\section{Discussion}

Dr Erle H. Austin III (Louisville, Ky). I would like to congratulate you on a clear presentation of new and important information that characterizes the neurologic condition of infants with severe CHDs when it is evaluated before they are subjected to the significant perturbations of bypass with or without DHCA.

I would also like to thank you for your involvement in this area. It is pediatric neurologists such as you that can help us identify the best ways to mitigate the adverse neurologic outcomes that continue to occur despite remarkable improvements in operative survival for infants with complex forms of congenital heart disease.
Your study, I think, is of value because it confirms a previously described high incidence of structural cerebral lesions in this group of patients. The most common lesion that you noted was PVL.

The major point of the work, however, relates to the low level of preoperative CBF that you have demonstrated with your technique of spin-label perfusion MRI. It is not difficult for us to accept this low level of CBF as a significant factor that might compromise the infant preoperatively and possibly make him or her more susceptible to additional neurologic injury with the operative procedure.

My major concern with the study is the use of a new technique for measuring $\mathrm{CBF}$ that does not appear to have been adequately compared with a more traditional technique, such as xenon washout. I recognize that such a comparison is not easy in newborn infants, but I am curious as to what studies have been done to validate this technique in an animal model or possibly clinical comparisons with transcranial Doppler scanning or other clinical measurement tools.

In addition, the values of CBF that you have shown are compared with normal values that are derived from a completely different technique of measuring $\mathrm{CBF}$.

This brings me to my first question.

Would you comment on the validity of spin-label perfusion MRI for accurately measuring $\mathrm{CBF}$ and how it has been compared with a gold standard. Also, could you comment on the absence of control data from a group of neonates without congenital heart disease.

Dr Licht. Thank you for your kind words.

Arterial spin labeling has been used for years in the adult stroke population and in functional MRI testing. It has been validated against $0^{15}$ positron emission tomography scans in adults in blood flow ranges between 20 and $80 \mathrm{~mL} \cdot 100 \mathrm{~g}^{-1} \cdot \mathrm{min}^{-1}$. A direct comparison with xenon-133 SPECT, however, has not been done.

Animal studies have been done in rats and larger mammals. That work was pioneered by John Detre, who is a coauthor of this article.

In functional MRI, PASL-pMRI has been directly compared with the blood oxygen level-dependent technique; however, the results of arterial spin labeling are more reproducible and stable over serial measurements. If regional $\mathrm{CBF}$ is measured during the performance of simple motor tasks in adult patients and then remeasured hours, days, or weeks later, the same values are obtained. The reproducibility of the results is very good.

The arterial spin-labeling measurements are also internally consistent: patients with the lowest CBF measurements have the highest risk for demonstrating PVL, presumably an ischemic injury. The reproducibility of our results has also been demonstrated by performing test-retest experiments under hypercarbic conditions. The reproducibility of our measurements $(9 \%)$ exceeded standard expectations (10\% error).

Dr Austin. How about using this in a set of control infants with that congenital heart disease? Do you want to comment about that?

Dr Licht. We have studied older children with arterial spin labeling to measure the developmental changes that take place in childhood. Our CBF values are essentially the same as those reported by Chiron and coworkers in their series in 1992 .

We have not measured neonates without CHD at this point. Our hospital only has our born neonates, and therefore these are not 
true normal control subjects. It is possible to study CBF with PASL-pMRI in infants without CHD who are receiving an MRI of the brain for other clinical indications. One must also keep in mind that we will never be able to study healthy neonates under the same study conditions (ie, intubated, paralyzed, and sedated).

Dr Austin. So what were the flow values that you were just referring to from Chiron and coworkers?

Dr Licht. Chiron and coworkers measured CBF in 42 children, ages 2 days to 19 years. The measurements were made for clinical neurologic indications that were retrospectively determined to be transient and unaccompanied by structural brain lesions. The children developed normally, and the SPECT measurements were added to the database of normal values.

Dr Austin. But what value of flow was measured? What was considered to be normal?

Dr Licht. The measurements were made in 7 neonates with a natural airway. The average value in these 7 neonates, ages 2 to 45 days, was $50 \pm 3.4 \mathrm{~mL} \cdot 100 \mathrm{~g}^{-1} \cdot \mathrm{min}^{-1}$.

Dr Austin. And clearly, in this group, these are significantly lower. And I think it's fair for us to assume that you've done a lot to try and validate this. Again, I think it's interesting and may be important data.

A second question relates to, there were 5 patients with very low preoperative cerebral blood flow measurements. Were there any clinical signs of a neurologic impairment in those patients?

Dr Licht. No.

Dr Austin. And recognizing the limitations of neurologic examinations in newborn babies. But you are a neurologist.

Dr Licht. Yes, there is no predictive value to the neonatal neurologic examination, and therefore neurologic examination was not part of this protocol. But these babies were all similar in that they had no prior history of birth asphyxia and no history of arrest of any sort.

Dr Austin. Finally, have you, at least anecdotally, used this technique postoperatively in any of the members of this cohort to determine whether there is any effect of the surgical procedure?

Dr Licht. We have not. But that study is in planning, and we should start recruitment in the next couple of weeks.

Christo I. Tchervenkov (Montreal, Quebec, Canada). I congratulate you for working in an area that I think is becoming increasingly important. It has been conventional wisdom to assign neurologic injury to what happens in the operating room or, perhaps, after the operating room. But we are beginning to recognize that some of these injuries might actually happen before any surgical insult.

A few years ago at the Montreal Children's Hospital, we did look at the neurologic status before surgical intervention in a series of neonates and infants with various diagnoses. To our surprise, $60 \%$ of neonates and $40 \%$ of infants had some kind of neurologic or developmental preoperative abnormalities. And in many of these, the abnormality persisted after the operation. There were very few new events.

And what was very interesting to see in that cohort of patients was that there seemed to be a difference between the cyanotic patients, such as transposition or tetralogy, and patients who had significant left-to-right shunts, such as complete atrioventricular canal, ventricular septal defect, hypoplastic left heart, and so on. And the incidence of preoperative abnormalities was higher in the noncyanotic patients, perhaps reflecting that in some of these diagnoses there is decreased CBF prenatally.

Therefore my question is the following: Have you looked at stratifying your cohort of patients when you are measuring $\mathrm{CBF}$ in terms of cyanotic patients versus noncyanotic patients?

Dr Licht. That is an excellent suggestion. We have not specifically compared CBF values in cyanotic versus noncyanotic groups. We have stratified the patients according to their clinical heart diagnosis. We have also classified them according to the method proposed by Clancy and colleagues in the risk-of-death article, in which heart lesions were grouped into one of 4 classes: single ventricle without arch obstruction, single ventricle with arch obstruction, 2 ventricles without arch obstruction, and 2 ventricles with arch obstruction. We also looked at the effect of the ductus arteriosus. We compared patients with a patent ductus arteriosus with those without, and determined whether the ductus was required for pulmonary circulation or systemic circulation. Thus far in our analysis, we have failed to find a significant correlation of CBF with any of the structural anatomic characteristics.

I will say that babies with a prenatal diagnosis of a CHD had significantly lower CBF values than babies given diagnoses at or after the time of birth. These babies did not differ significantly in birth weight or head circumference. Furthermore, although most babies with hypoplastic left heart syndrome were given diagnoses prenatally and babies with transposition of the great arteries were given diagnoses postnatally, the babies with transposition of the great arteries had much lower CBF values than babies with hypoplastic left heart syndrome. Whether this is a spurious result remains to be seen after further evaluation.

Dr Marshall L. Jacobs (Philadelphia, Pa). This is a lovely study, and you obviously found the issue of PVL sufficiently significant to single it out with the yellow bars and highlight it in multiple aspects of the analysis. Of course, the same group with, I guess, Dr Gaynor as the author documented a certain frequency in preoperative patients, some evolution, and some new findings of PVL in postoperative patients. Could you share with the group your insights as to what specifically is the significance of this particular finding and how closely it correlates with inadequate blood flow and hypoxic injury.

Dr Licht. The injury itself, PVL, is believed to arise from several factors in premature babies. One major contributor is the immature oligodendrocyte. In the developing brain these cells are highly metabolically active and are particularly vulnerable to hypoxic ischemic injury. Another consideration is that the injury occurs in the watershed region between the small arteries that penetrate from the cortex and those that arise from the ventricle surface and run radially outward. This watershed area is especially prone to ischemia during decreases in blood pressure. There is also a growing suspicion that immature white matter might be injured by the systemic inflammatory response triggered by exposure to bypass. It is the combination of these factors that might lead to PVL, and this was the basis of our study and why we decided to study CBF.

Dr Ross M. Ungerleider (Portland, Ore). Certainly The Children's Hospital of Philadelphia, with you and with the addition of Bill Gaynor and Bill Greeley, who worked with me at Duke for so many years, is really becoming a leading center in helping us understand more about cerebral injury in infants. 
Just listening to your data, it strikes me that you probably could go back and do a calculation, and I would be interested to hear whether you have, and that would be cerebral oxygen delivery. You know what the hematocrit values are of the patients that you are studying, you know what their blood oxygen levels are, and now you know the CBF. It would be interesting to know whether the cerebral oxygen delivery in any way correlated with some of the findings that you had in terms of neurologic events.
Dr Licht. Well, we have collected a lot of data but have not completed the calculations for oxygen delivery. The placement of venous and arterial central lines was not dictated by the study protocol, and therefore not all patients had lines at the time of the study. Furthermore, the location of the central venous lines needs to be confirmed by reviewing the chest radiographs performed at the time of the study to ascertain the accuracy of the mixed venous measurements. These data will be available soon.

\section{ON THE MOVE?}

\section{Send us your new address at least six weeks ahead}

Don't miss a single issue of the journal! To ensure prompt service when you change your address, please photocopy and complete the form below.

Please send your change of address notification at least six weeks before your move to ensure continued service. We regret we cannot guarantee replacement of issues missed due to late notification.

\section{JOURNAL TITLE:}

Fill in the title of the journal here.

\section{OLD ADDRESS:}

Affix the address label from a recent issue of the journal here.

\section{NEW ADDRESS:}

Clearly print your new address here.

Name

Address

City/State/ZIP
COPY AND MAIL THIS FORM TO:

Elsevier Inc.

Subscription Customer Service

6277 Sea Harbor Dr

Orlando, FL 32887
OR FAX TO:

407-363-9661

OR E-mail:

elspcs@elsevier.com
OR PHONE:

800-654-2452

Outside the U.S., call

407-345-4000 